\title{
The language of obesity just makes matters worse
}

\author{
Obesity prevention has emphasized the individual person and created a narrative of blame. \\ But by treating obesity as a socially transmitted disease, we can start to turn the tide of the \\ obesity epidemic, says Tim Lobstein.
}

( ver 700 million people currently have a preventable disease: obesity. But the way we describe obesity and its causes, and especially how we portray the people who live with obesity, could make matters worse. The language we use diverts our attention from the root causes, misleads our efforts to prevent obesity and makes it harder for people to lose weight.

Let me explain. In what we can now see as the early days of the obesity epidemic, in the late 1970s, the UK government became aware of the rising levels of chronic disease in the population and asked the government-funded Health Education Council to produce some advice. In 1979, the council's classic health education booklet Look After Yourself! suggested, "A practical way to prevent obesity is not to become overweight." We may laugh at the ineffectual advice now, but it encapsulated the prevailing assumption in many societies that people who became obese had a choice, that they allowed themselves to become overweight, that they failed to prevent excess weight gain and indeed failed to look after themselves'. By giving such advice, the authorities had done what they could. The rest was up to us.

We have moved on from those days in several ways. Obesity rates have risen from less than $4 \%$ of UK adults in the 1970 s to $27 \%$ in 2015 ; a similar pattern is seen in Western Europe, the United States and Australia, and obesity rates are now even higher in emerging economies in the Middle East and parts of Central America. While governments continue to invest in health education campaigns, they are also recognizing that these are insufficient to reverse the epidemic. Additional measures are now seen as essential, including market interventions, such as restrictions on advertising junk food to children on TV and on the Internet, taxes and levies on sugarladen soft drinks, front-of-pack nutrition labelling, and sugar and calorie reduction targets for the food industry. These measures sit alongside moves to restrict sales of junk food in hospitals, improved school meal standards and moves to have calorie labelling on menus in fast food outlets.

But there is still some way to go to achieve a more appropriate narrative about responsibility and to undo the harm that the emphasis on the individual is doing. First, we should review the use of the term 'non-communicable disease' to describe obesity and its complications, including the increased risk of diabetes, liver disease, cardiovascular disease and several common cancers. These diseases might not be spread by bacterial or viral vectors but they are communicated in a social sense: through family behaviour patterns, through institutional food supply policies, and especially through the commercial promotion of unhealthy food and sedentary behaviour. These vectors powerfully amplify the body's biological urge to store energy and avoid effort, and transmit the risk of obesity from parent to child, from neighbourhood to family, from producer to consumer. This makes obesity a socially communicable disease.

\section{We should review the use of the term 'non-communicable disease' to describe obesity and its complications.}

The focus on individual responsibility suits commercially interested parties both those who sell obesogenic products and those who sell ineffective solutions - and also suits governments unwilling to regulate the marketplace. But it has created a narrative of blame that pervades the popular media. Take a careful look at the images that accompany media stories about obesity: they have for many years typically shown people with extreme levels of obesity, usually badly dressed, often consuming junk food or lying on sofas, and frequently without their faces showing. They are depersonalized images encouraging disgust. We don't do this with cancer, diabetes or heart disease, so why with obesity?

This narrative is absorbed by the health professionals who offer treatment for obesity and its complications. Several surveys have shown that a significant proportion of doctors, nurses and clinic staff hold obese people to be responsible for their condition, and many say they find it hard to start a conversation with a patient with obesity, and some admit they find it hard to physically touch a person with morbid obesity. Further, this 'fat shaming' narrative of personal responsibility for a socially transmitted disease is absorbed and believed by people living with obesity. Such internalized shame and self-critical stigma is itself harmful: it adds to the risk of depression and has been shown to reduce the likelihood of seeking weight-loss treatment, and to reduce the likelihood of success when such treatment is tried.

We have made great strides in reducing tobacco-related diseases, and we are starting to turn the tide on the harmful use of alcohol. Obesity needs a similar approach and an even greater change in attitudes, but at present the language and images of individual blame and shame are just making matters worse.

\section{Tim Lobstein ${ }^{1,2}$ \\ ${ }^{1}$ World Obesity Federation, London, UK. ${ }^{2}$ The Boden Institute, University of Sydney, Sydney, NSW, Australia. \\ e-mail: tlobstein@worldobesity.org}

Published online: 5 February 2018 https://doi.org/10.1038/s41562-018-0295-6

Competing interests

The author declares no competing interests. 